\title{
A importância da enof talmia senil no desenvolvimento do entrópio involucional
}

\author{
The role of senile enophthalmos in involutional entropion
}

\author{
Nilson Lopes da Fonseca Junior ${ }^{1}$ \\ Lúcia Miriam Dumont Lucci ${ }^{2}$ \\ José Ricardo Carvalho Lima Rehder ${ }^{3}$
}

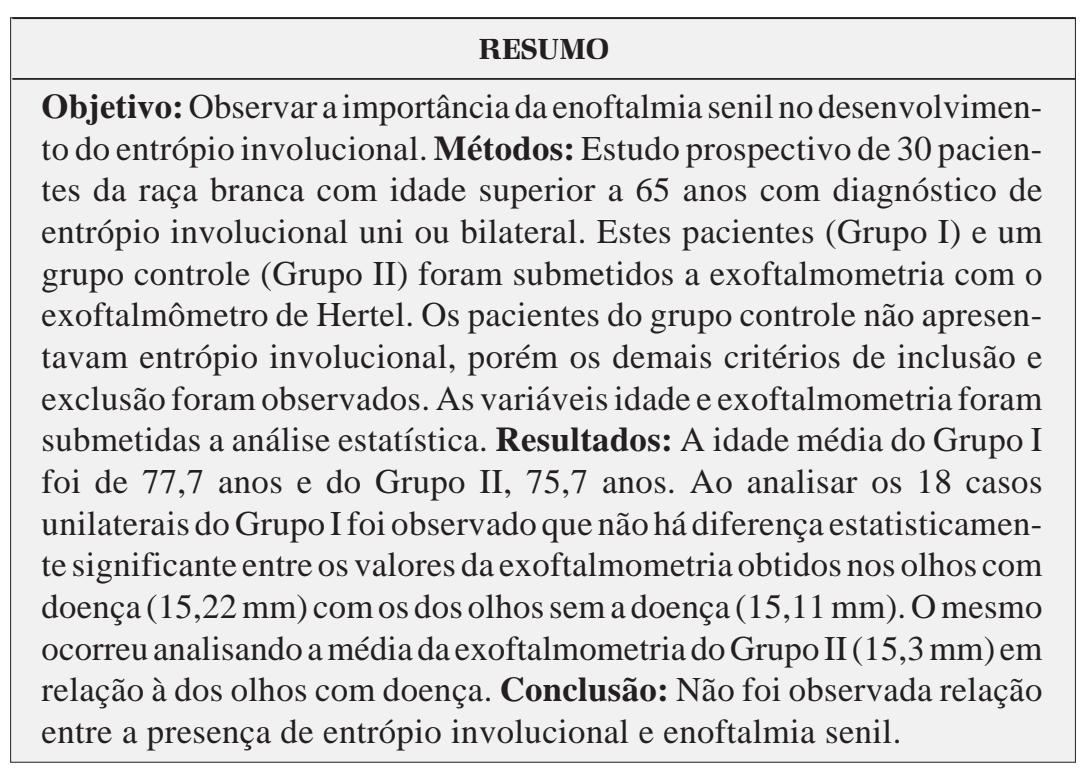

Descritores: Entrópio/cirurgia; Enoftalmia; Pálpebras/cirurgia; Exoftalmia; Técnicas de diagnóstico oftalmológico
Trabalho realizado na Disciplina de Oftalmologia da Faculdade de Medicina do ABC.

${ }^{1}$ Médico colaborador do Setor de Plástica Ocular e Órbita da Disciplina de Oftalmologia da Faculdade de Medicina do ABC - FMABC - Santo André (SP) - Brasil.

${ }^{2}$ Chefe do Setor de Plástica Ocular da Disciplina de Oftalmologia da FMABC - São Bernardo do Campo (SP) - Brasil.

${ }_{3}^{3}$ Professor Titular da Disciplina de Oftalmologia da FMABC - São Bernardo do Campo (SP) - Brasil.

Endereço para correspondência: Nilson Lopes da Fonseca Junior. Rua Antonio Canero, 27 - São Paulo (SP) CEP 03190-140

E-mail: lopesdafonseca@uol.com.br

Recebido para publicação em 19.04.2005

Última versão recebida em 22.12.2005

Aprovação em 01.08.2006

Nota Editorial: Depois de concluída a análise do artigo sob sigilo editorial e com a anuência dos Drs. Simone H. D. Von Faber Bison e Roberto Lorens Marback sobre a divulgação de seus nomes como revisores, agradecemos sua participação neste processo.

\section{INTRODUÇ̃̃OO}

O entrópio é uma alteração anatômica da pálpebra caracterizada pela inversão da margem ${ }^{(1)}$. Ocasiona irritação ocular, sensação de corpo estranho, lacrimejamento, ceratite e ulceração corneana devido ao trauma constante dos cílios contra o globo ocular ${ }^{(1-2)}$. O entrópio adquirido pode ser classificado em: cicatricial e senil ${ }^{(2)}$.

O entrópio involucional é o mais freqüente e acomete exclusivamente a pálpebra inferior. A fisiopatologia é explicada pelos seguintes fatores: 1) frouxidão horizontal da pálpebra inferior, atrofia do tarso e frouxidão do tendão cantal lateral e medial; 2) diminuição da tensão vertical da pálpebra inferior pela frouxidão dos retratores da pálpebra inferior e do septo orbitário; 3) sobreposição do feixe pré-septal do músculo orbicular palpebral sobre o feixe pré-tarsal $\left.{ }^{(1-8)} ; 4\right)$ enoftalmia senil secundária a atrofia da gordura orbitária ${ }^{(6,9-10)}$.

Algumas técnicas cirúrgicas para o tratamento do entrópio involucional foram desenvolvidas, sendo todas direcionadas para a correção das alterações anatômicas responsáveis por esta alteração, incluindo o encurtamento da pálpebra inferior, a reinserção dos músculos retratores da pálpebra inferior e o redirecionamento do feixe pré-septal do músculo orbicular ${ }^{(1,4-8)}$.

O primeiro relato da correção cirúrgica do entrópio senil data no século I, 
quando Celsus sugeriu uma incisão cutânea e do músculo orbicular $^{(5)}$. Em 1972, Jones et al., descreveram a técnica de reinserção dos músculos retratores da pálpebra inferior no tarso, objetivando restabelecer a anatomia da pálpebra inferior. Este procedimento ainda é o procedimento de escolha para o tratamento do entrópio senil ${ }^{(11)}$.

O entrópio pode estar associado a microftalmia ou anoftalmia, porém a contribuição da enoftalmia senil para o desenvolvimento do entrópio involucional é controversa na literatura ${ }^{(2,5)}$.

A enoftalmia é o deslocamento do bulbo ocular para o interior da cavidade orbitária, apresentando uma diminuição na exoftalmometria superior a $2 \mathrm{~mm}^{(12)}$.

Atualmente, o método mais utilizado para se diagnosticar qualquer alteração no posicionamento do bulbo ocular em relação a cavidade orbitária é realizado pelo exoftalmômetro de Hertel. Este equipamento apresenta um sistema de espelhos que possibilita a exoftalmometria de ambos os olhos simultaneamente. $\mathrm{O}$ exame é realizado após o posicionamento do instrumento, apoiando-o nas rimas laterais orbitárias, sendo solicitado para o paciente que se mantenha em posição primária do olhar ${ }^{(13-15)}$.

A exoftalmometria obtida por este instrumento representa a distância entre o ápice da córnea e a rima da parede lateral orbitária. Uma outra medida denominada base pode ser obtida e representa a distância entre as rimas laterais orbitárias, e deve ser mantida nas exoftalmometrias no mesmo paciente ${ }^{(14-15)}$.

O objetivo deste trabalho é observar a importância da enoftalmia senil no desenvolvimento do entrópio involucional.

\section{MÉTODOS}

Estudo prospectivo de 30 pacientes com diagnóstico de entrópio involucional do Ambulatório de Plástica Ocular da Disciplina de Oftalmologia da Faculdade de Medicina do ABC, após a aprovação pelo Comitê de Ética em pesquisa da instituição. Foram incluídos pacientes da raça branca com idade superior a 65 anos e portadores de entrópio senil uni ou bilateral.

Os critérios de exclusão foram: 1) história prévia de cirurgia palpebral e/ou orbitária; 2) outra doença palpebral e/ou orbitária; 3) pacientes da raça amarela e negra; 4) antecedentes pessoais de doenças corneanas como ceratocone, ceratopatia pelúcida; 5) pacientes com alteração no diâmetro antero-posterior do globo ocular como por exemplo alta miopia, phthisis bulbi, buphthalmos, microftalmo; 6) pacientes com incapacidade de compreender o estudo.

Todos os 30 pacientes portadores de entrópio involucional (Grupo I) foram submetidos a exoftalmometria com o exof- talmômetro de Hertel. O paciente permanecia sentado e o exoftalmômetro de Hertel era apoiado na rima orbitária lateral. O examinador observava a medida da exoftalmometria de cada olho do paciente.

No grupo controle (Grupo II), formado por 30 pacientes normais, também foi realizada a exoftalmometria de Hertel. Os pacientes deste grupo não apresentavam entrópio involucional, porém os demais critérios de inclusão e exclusão foram observados.

\section{MÉTODO ESTATÍSTICO}

Inicialmente as variáveis foram analisadas descritivamente. Para as variáveis quantitativas (idade e exoftalmometria) esta análise foi realizada através da observação dos valores mínimos e máximos, e do cálculo de médias, medianas e desvios-padrão.

Para a comparação entre a média dos dois grupos (com e sem doença) foi utilizado o teste $t$ de Student para amostras independentes ${ }^{(16)}$.

Todos os testes foram realizados admitindo-se o nível de significância de $5 \%$.

\section{RESULTADOS}

Foram avaliados 60 pacientes, dos quais 30 apresentavam a doença em estudo (Grupo I) e 30 sem a doença (Grupo II). Dos 30 pacientes do Grupo I, 33,4\% (10 pacientes) apresentavam doença no olho direito, $26,6 \%$ (8 pacientes) no olho esquerdo, e 40,0\% (12 pacientes) em ambos os olhos.

A idade dos pacientes do Grupo I variou de 66 a 90 anos (média de 77,7 anos) e do Grupo II variou de 64 a 100 anos (média de 75,7 anos). Observamos na tabela 1 que os grupos não diferem em relação à idade.

Os pacientes pertencentes ao Grupo II (controle) apresentaram uma exoftalmometria média de 15,13 mm (Tabela 2).

Ao serem analisados os 18 casos unilaterais do Grupo I observou-se que não há diferença estatisticamente significan-

\begin{tabular}{|c|c|c|c|}
\hline \multirow[b]{2}{*}{ Variável } & \multicolumn{2}{|c|}{ Grupo } & \multirow[b]{2}{*}{$p$} \\
\hline & Estudo & Controle & \\
\hline Idade & $77,77 \pm 8,77$ & $75,73 \pm 6,59$ & $0,314^{*}$ \\
\hline
\end{tabular}

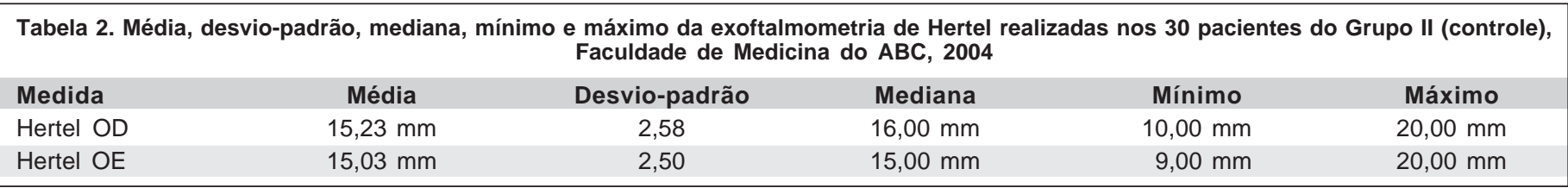


te entre os valores da exoftalmometria de Hertel obtidos nos olhos com doença e nos olhos sem a doença (Tabela 3).

Dos 120 olhos (60 pacientes) avaliados neste estudo, 78 $(65,0 \%)$ não apresentavam doença (Grupo controle +18 casos unilaterais) e $42(35,0 \%)$ com doença (12 casos bilaterais +18 casos unilaterais). Ao serem analisados os valores da exoftalmometria de Hertel, observou-se que os olhos com e sem a doença não apresentam uma diferença estatisticamente significante (Tabela 4$)$.

\section{DISCUSSÃO}

O entrópio senil é uma alteração no posicionamento palpebral comum na população idosa ${ }^{(2)}$.

A presença da enoftalmia senil, apesar de ser considerada como um fator para desenvolvimento do entrópio involucional em alguns estudos ${ }^{(2,9-10)}$, não parece estar bem estabelecida na literatura.

Algumas situações específicas nas quais o deslocamento posterior do globo ocular poderia estar associado ao desenvolvimento do entrópio, porém nestes casos são observadas outras alterações orbitárias ou oculares, como por exemplo, fraturas orbitárias e microftalmia ${ }^{(17)}$. Estas afirmações não podem ser consideradas neste estudo, pois nos casos de entrópio involucional a cavidade orbitária e o globo ocular apresentam-se íntegros.

Alguns autores justificam o desenvolvimento da enoftalmia senil baseado na atrofia da gordura orbitária e frouxidão dos tecidos orbitários, e tentam relacioná-la ao aparecimento do entrópio involucional ${ }^{(9,17)}$.

Outros autores compararam a exoftalmometria de dois grupos, sendo um grupo composto por pacientes normais com mais de 65 anos de idade e outro, composto por pacientes normais entre 15 e 44 anos de idade. Exoftalmometrias discretamente menores no primeiro grupo foram observadas, o que poderia caracterizar uma enoftalmia senil ${ }^{(18)}$.

Alguns autores obtiveram a exoftalmometria em uma população de 681 adultos entre 18 e 91 anos de idade. Estes autores não observaram a presença de enoftalmia senil, definindo uma exoftalmometria média de $16,5 \mathrm{~mm}$ para homens brancos e $15,4 \mathrm{~mm}$ para mulheres brancas em qualquer idade ${ }^{(19)}$. No atual estudo a exoftalmometria média do grupo controle foi de 15,13 $\mathrm{mm}$.

Outros autores realizaram um estudo onde a exoftalmometria média no grupo que apresentava entrópio senil foi de $16 \mathrm{~mm}$ e no grupo controle, $16,15 \mathrm{~mm}$; ou seja, não observaram relação entre a enoftalmia senil e o entrópio involucional ${ }^{(2)}$.

\section{CONCLUSÃO}

No presente estudo não foi observada uma relação entre a presença de entrópio involucional e enoftalmia senil.

\section{ABSTRACT}

Purpose: To observe the role of senile enophthalmos in involutional entropion. Methods: Prospective clinical study comparing a population of 30 white patients above 65 years with involutional entropion (Group I) of an age- and race-matched control group (Group II). All age and exophthalmometric values were analyzed by a statistical study. Results: The average age was 77.7 years in Group I and 75.7 years in Group II. Eyes with involutional entropion are no more likely to have enophthalmos $(15.22 \mathrm{~mm})$ than is the involved contralateral eye $(15.11 \mathrm{~mm})$ or normal eyes of an age- and race-matched control group $(15.13 \mathrm{~mm})$. Conclusion: There is no correlation between involutional entropion and senile enophthalmos.

Keywords: Entropion/cirurgia; Enophthalmos; Eyelids/surgery; Exophthalmus; Diagnostic techniques, ophthalmological

\section{REFERÊNCIAS}

1. Siegel RJ. Involutional entropion: a simple and stable repair. Plast Reconstr Surg. 1988;82(1):42-7. Comment in: Plast Reconstr Surg. 1989;83(2):392-4.

2. Kersten RC, Hammer BJ, Kulwin DR. The role of enophthalmos in involutional entropion. Ophthal Plast Reconstr Surg. 1997;13(3):195-8.

3. Hurwitz JJ. Senile entropion: the importance of eyelid laxity. Can J Ophthalmol. 1983;18(5):235-7.

4. Rainin EA. Senile entropion. Arch Ophthalmol. 1979;97(5):928-30.

\begin{tabular}{|c|c|c|c|c|c|}
\hline Olho & Média & Desvio-padrão & Mediana & Mínimo & Máximo \\
\hline Com doença & $15,22 \mathrm{~mm}$ & 3,25 & $16,00 \mathrm{~mm}$ & $8,00 \mathrm{~mm}$ & $20,00 \mathrm{~mm}$ \\
\hline Sem doença & $15,11 \mathrm{~mm}$ & 3,55 & $15,50 \mathrm{~mm}$ & $7,00 \mathrm{~mm}$ & $20,00 \mathrm{~mm}$ \\
\hline
\end{tabular}

\begin{tabular}{|c|c|c|c|c|c|c|}
\hline Olho & $\mathbf{n}$ & Média & Desvio-padrão & Mediana & Mínimo & Máximo \\
\hline Sem doença & 78 & $15,13 \mathrm{~mm}$ & 2,77 & $15,50 \mathrm{~mm}$ & $7,00 \mathrm{~mm}$ & $20,00 \mathrm{~mm}$ \\
\hline Com doença & 42 & $14,02 \mathrm{~mm}$ & 3,33 & $15,00 \mathrm{~mm}$ & $8,00 \mathrm{~mm}$ & $20,00 \mathrm{~mm}$ \\
\hline
\end{tabular}


5. Nowinski TS. Orbicularis oculi muscle extirpation in a combined procedure for involutional entropion. Ophthalmology. 1991;98(8):1250-6.

6. Van den Bosch WA, Rosman M, Stijnen T. Involutional lower eyelid entropion: results of a combined approach. Ophthalmic Surg Lasers. 1998;29(7):581-6.

7. Danks JJ, Rose GE. Involutional lower lid entropion: to shorten or not to shorten? Ophthalmology. 1998;105(11):2065-7. Comment in: Ophthalmology. $1999 ; 106(5): 859$.

8. Cook T, Lucarelli MJ, Lemke BN, Dortzbach RK. Primary and secondary transconjunctival involutional entropion repair. Ophthalmology. 2001;108(5):989-93.

9. Dalgleish R, Smith JL. Mechanics and histology of senile entropion. Br J Ophthalmol. 1966;50(2):79-91.

10. Benger RS, Frueh BR. Involution entropion: a review of the management. Ophthalmic Surg. 1987;18(2):140-2.

11. Jones LT, Reeh MJ, Wobig JL. Senile entropion. A new concept for correction. Am J Ophthalmol. 1972;74(2):327-9.

12. Bite U, Jackson IT, Forbes GS, Gehring DG. Orbital volume measurements in enophthalmos using three-dimensional CT imaging. Plast Reconstr Surg. 1985;75(4):502-8.
13. Sleep TJ, Manners RM. Interinstrument variability in Hertel-type exophthalmometers. Ophthal Plast Reconstr Surg. 2002;18(4):254-7.

14. Kratky V, Hurwitz JJ. Hertel exophthalmometry without orbital rim contact. Ophthalmology. 1994;101(5):931-7. Comment in: Ophthalmology. 1995;102 (1):2-3; author reply 4. Ophthalmology. 1995;102(1):2; author reply 4. Ophthalmology. 1995;102(1):3-4.

15. Cole HP 3rd, Couvillion JT, Fink AJ, Haik BG, Kastl PR. Exophthalmometry: a comparative study of the Naugle and Hertel instruments. Ophthal Plast Reconstr Surg. 1997;13(3):189-94. Comment in: Ophthal Plast Reconstr Surg. 1999;15(4):299-301.

16. Rosner B. Fundamentals of Biostatistics. $2^{\text {nd }}$ ed. Boston: PWS; 1986.

17. Jones LT. The anatomy of the lower eyelid and its relation to the cause and cure of entropion. Am J Ophthalmol. 1960;49:29-36.

18. Frueh BR, Musch DC, Garber FW. Exophthalmometer readings in patients with Graves' eye disease. Ophthalmic Surg. 1986;17(1):37-40.

19. Migliori ME, Gladstone GJ. Determination of the normal range of exophthalmometric values for black and white adults. Am J Ophthalmol. 1984;98(4): 438-42.

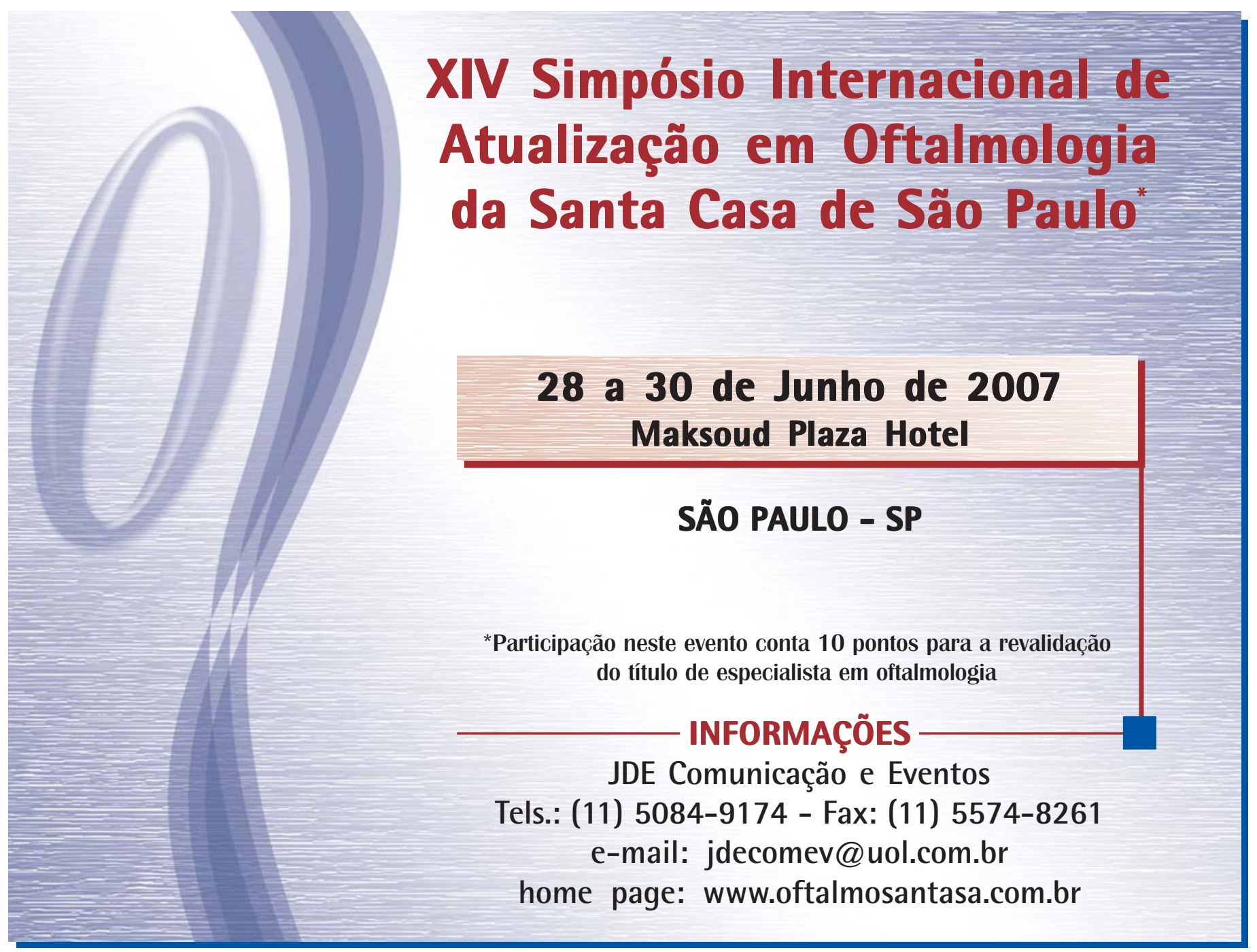

